\title{
Dark Matter Detector Proves its Sensitivity
}

\author{
A new sensor provides world-leading sensitivity for distinguishing \\ lightweight dark matter from background noise.
}

\section{By Rachel Berkowitz}

$\Lambda$ mong the models researchers have come up with to explain dark matter, one proposes that dark matter consists of tiny particles with masses below $1 \mathrm{GeV} / c^{2}$. These theoretical particles are so lightweight that conventional efforts based on nuclear recoil could never detect them. Instead, researchers hunt them by searching for evidence of their interactions with electrons in silicon detectors. Now the SENSEI Collaboration has demonstrated such a detector designed to spot lightweight dark matter with record-breaking sensitivity. The finding could provide a valuable tool in astroparticle physics.

A sub-GeV dark matter particle's energy should excite one or several electrons when the particle strikes a silicon charge-coupled device. These detectors are common in digital imaging, but the SENSEI device differs in that it measures precisely how many electrons are excited in each pixel. The challenge when deploying such a sensitive instrument is in distinguishing electron excitations caused by dark matter from those caused by other background processes.

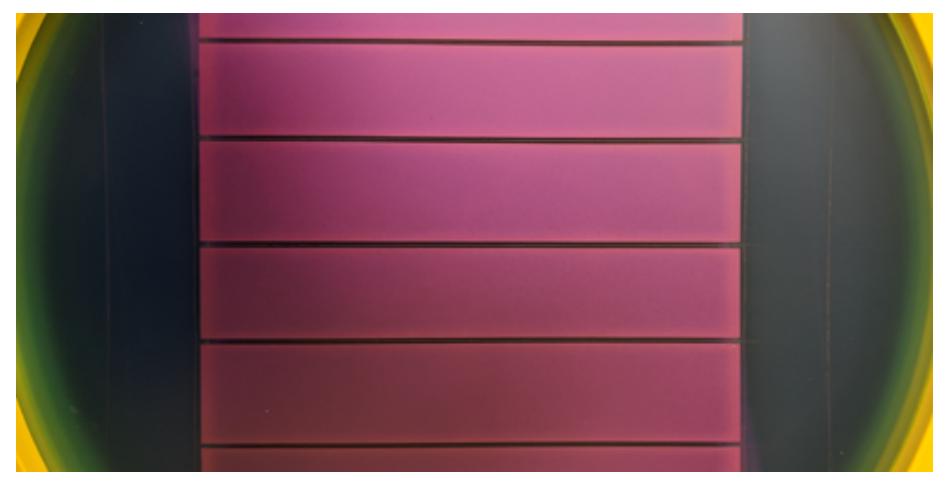

Credit: SENSEI Collaboration
To determine how well their 5.4-megapixel detector could pick out a potential dark matter signal, the SENSEI team placed it in a shielded underground chamber. They found that the observed rate of single-electron events correlates with the shield thickness and, hence, the rate of background events that affect the detector. This finding suggests that many observed events originate from background sources. The researchers calculated upper limits for how many electrons per pixel per day could be attributed to different lightweight dark matter particles-improving on previous detector limits by orders of magnitude for certain particles.

Next, the team will deploy multiple sensors inside a well-shielded environment at the SNOLAB neutrino observatory. That design will be sensitive to even lighter dark matter particles.

Rachel Berkowitz is a Corresponding Editor for Physics based in Seattle, Washington, and Vancouver, Canada.

\section{REFERENCES}

1. L. Barak et al. (SENSEI Collaboration), "SENSEI: Direct-detection results on sub-GeV dark matter from a new Skipper CCD," Phys. Rev. Lett. 125, 171802 (2020). 